\title{
Beherrschung stark korrelierter Logistik- und Produktions-Prozesse
}

\author{
Handling of Strongly Correlated Logistics and Production Processes
}

\author{
Sebastian Rank* \\ Tobias Uhlig ** \\ Thorsten Schmidt * \\ Oliver Rose ** \\ * Professur für Technische Logistik \\ Institut für Technische Logistik und Arbeitssysteme \\ Technische Universität Dresden \\ **Professur für Modellbildung und Simulation \\ Institut für Technische Informatik \\ Universität der Bundeswehr München
}

B ei der Simulation von Logistik- und Produktionssystem werden Zufallszahlengeneratoren verwendet, um stochastische Einflüsse zu modellieren. Ein wichtiges Qualitätsmerkmal dieser Generatoren ist die Erzeugung möglichst unabhängiger Zufallszahlen. Werden jedoch reale Prozesse betrachtet, so sind die Daten im Allgemeinen nicht unabhängig. Diese Arbeit befasst sich mit der Analyse von Praxisdaten bezüglich des Auftretens von Abhängigkeiten. Dazu werden Korrelationsstrukturen gesucht. Außerdem wird gezeigt, dass unabhängige Zufallszahlen in der Regel ungeeignet sind, um stochastische Prozesse mit ausgeprägten Abhängigkeiten zu modellieren.

[Schlüsselwörter: Autokorrelation, Simulation, Zufallszahlengenerator, Ankunftsprozessmodellierung]

$\mathbf{R}$ andom number generators are widely used to model stochastic processes in logistics and production systems. Creating truly independent random numbers is one important feature of these generators. However, actual data in real world systems is rarely independent. This paper discusses occurrences of dependencies in observed data by examining sample data for correlation structures. It will be demonstrated that independent random numbers created by common generators are not suitable to model processes with distinct dependencies.

[Keywords: autocorrelation, simulation, random number generator, modeling of arrival processes]

\section{EINLEITUNG}

Seit Jahren wird in der einschlägigen Literatur dargelegt, dass die Komplexität von Logistik- und Produktionssystemen stetig zunimmt [Zae02]. Um den Herausforderungen der Planung und Steuerung gerecht $\mathrm{zu}$ werden, wird unter anderem verstärkt auf das Werkzeug der Ablaufsimulation zurückgegriffen [VDI10; Kud00; LK00]. Auf diese Weise ist es möglich, über eine deterministische Sichtweise hinaus stochastische Aspekte dynamisch abzubilden. Das Eintreten von Ereignissen erfolgt dabei scheinbar zufällig. In gängigen Softwareprodukten wird bei der Erzeugung der Ereignisse viel Wert darauf gelegt, dass diese unabhängig voneinander auftreten [Lec06]. Bereits einfache Überlegungen, wie z. B. Losbildung oder das Beachten von Prioritätsregeln bei der Auftragseinlastung, führen aber zum Schluss, dass aufeinanderfolgende Vorgänge nicht zwangsläufig unabhängig voneinander sind, sondern korrelieren. Wird dieser Umstand übersehen, führt dies gegebenenfalls zu Modellen, welche keiner Validierung standhalten und verfälschte Ergebnisse liefern. Die Folgen für die Auslegung und Steuerung von Anlagen sind weitreichend [VDI10].

Eine wesentliche Aufgabe der Modellierung von Logistik- und Produktionssystemen besteht in der Beschreibung der Prozessscharakteristik, wie etwa schwankende Bedienzeiten einer Servicestation oder unregelmäBiges Auftreten von Forderungen. Die Parametrierung von Modellelementen oder die Beschreibung der Eintrittswahrscheinlichkeit von Ereignissen basiert dabei in der Regel auf Realdaten. Häufig werden Modelle jedoch zur Simulation eines Neu- oder Umbauprojektes verwendet. Dann wird es nötig, entsprechende Parameter und Daten zu schätzen und passende Verteilungsfunktionen zu wählen. So ist es z. B. üblich, eine Exponentialverteilung zur Beschreibung von Zwischenankunftszeiten von Lieferfahrzeugen heranzuziehen [LK00].

Die Erfahrung der Autoren zeigt, dass vor allem Projektpartner aus dem praxisnahen Umfeld oft zu oberflächlich bei der Auswahl und Parametrierung einer Verteilungsfunktion vorgehen. Der Fokus wird meist zu stark allein auf den Mittelwert $(\mu)$ gelegt. Bestenfalls ist die 
Auswirkung einer hohen Schwankung/ Standardabweichung $(\sigma)$ der zugrundeliegenden Verteilung auf das Systemverhalten bekannt (vgl. Abbildung 1).

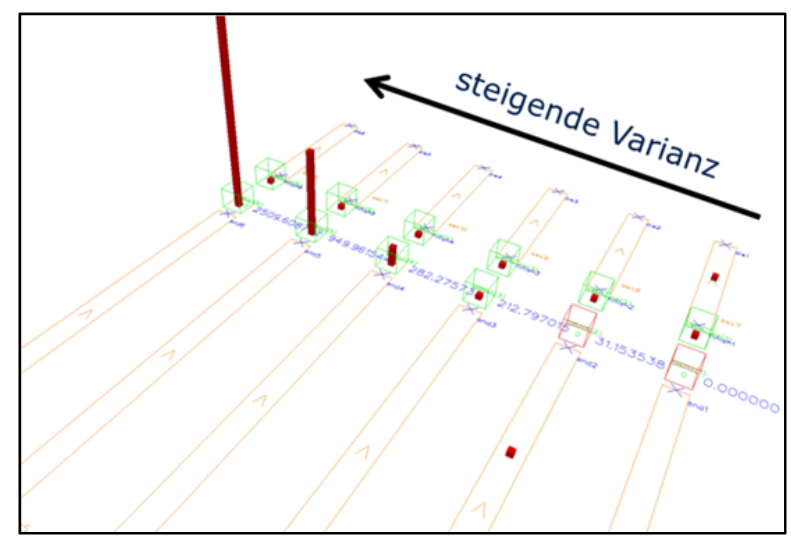

Abbildung 1. Simulation von M/D/1-Modellen mit identischem mittleren Ankunftsabstand und identischer Verteilungsfunktion für alle Bahnen. Steigende Varianz (Variationskoeffizient $\sigma / \mu$ ) führt zu längeren Warteschlangen

Zusammenfassend kann festgehalten werden, dass zufällige Ereignisse in logistischen Simulationsmodellen durch drei Eigenschaften charakterisiert werden: Eine zugrundeliegende Verteilung, deren Mittelwert und deren Varianz. Dies ist der Stand der Technik, der heutzutage als geeignete Modellierung akzeptiert wird. Die eingangs erwähnten Beispiele bzgl. Prioritätsregeln oder Losbildung werfen jedoch die Frage auf, ob dies ausreichend ist. Folgendes kleines Produktionsmodell (vgl. Abbildung 2) und zugehöriges Simulationsmodell (vgl. Abbildung 3) illustrieren die Defizite einer solchen Modellierung.

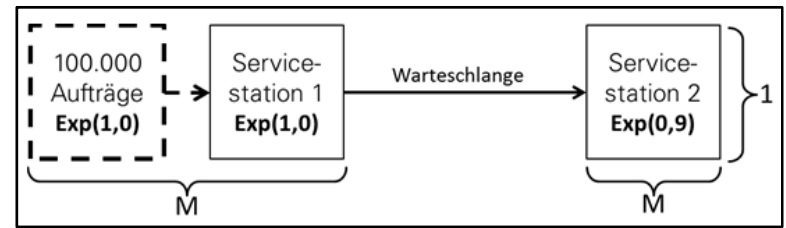

Abbildung 2. Produktionsmodell

Es sollen 100.000 Aufträge zunächst in Servicestation 1 bearbeitet werden. Jeder Auftrag verweilt eine spezifische Bearbeitungszeit in Servicestation 1. Diese ist exponentialverteilt mit Parameter $\lambda=1 \rightarrow \operatorname{Exp}(1,0)$. Der zweite Arbeitsschritt erfolgt in Servicestation 2. Die Bearbeitungszeit ist so gewählt, dass eine Auslastung von $90 \%$ erreicht wird. Die Bearbeitung erfolgt wiederum exponentialverteilt. Es ergibt sich so $\lambda=1 * 0,9 \rightarrow \operatorname{Exp}(0,9)$. Ausgehend vom Abgangsstrom der Servicestation 1 entspricht das Modell nach Kendall-Notierung einem M/M/1-System [Ken53]. Für das erste Experiment wird der oben beschriebene Aufbau ohne weitere Annahmen simuliert. Für den zweiten Versuch wird eine Priorisierung für Batches von Aufträgen angewandt, d. h. in Blöcken von je 100 Aufträgen sollen zunächst diejenigen Positionen mit einer kürzeren Bearbeitungszeit priorisiert werden. Im dritten Versuch soll die Priorisierung kurzer Bearbeitungszeiten über alle 100.000 Aufträge erfolgen, die Aufträge werden also der Bearbeitungszeit nach sortiert (Kendall-Notierung bei Priorisierung: G/M/1). Die Ergebnisse sind in Abbildung 3 und Tabelle 1 dargestellt.

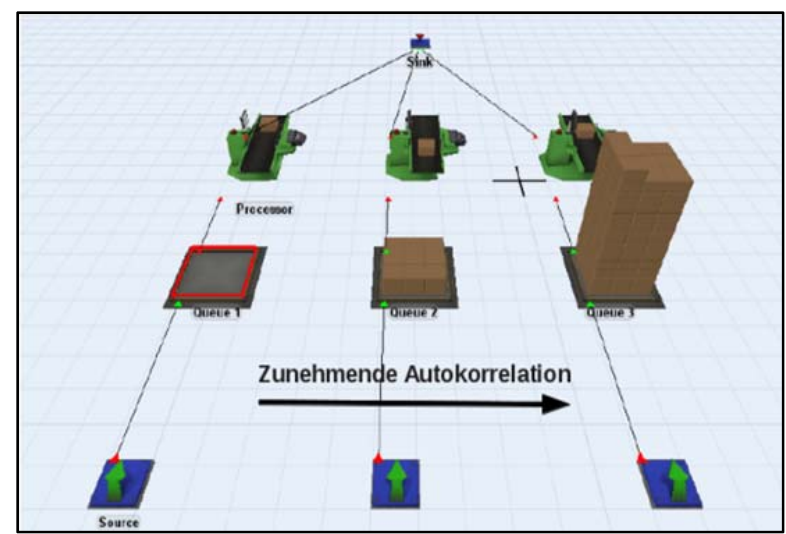

Abbildung 3. Simulation von M/M/1 bzw. G/M/1 - Modellen. Autokorrelierte Zwischenankunftszeiten führen bei sonst identischer Verteilung zu längeren Warteschlangen

Tabelle 1. Ergebnisse der Auswirkung von Batchbildung und Prioritätsregeln bei der Auftragseinlastung

\begin{tabular}{|l|r|r|r|}
\hline Servicestation 2 & Versuch 1 & Versuch 2 & Versuch 3 \\
\hline $\begin{array}{l}\text { Ø Warteschlange } \\
\text { [\# Aufträge] }\end{array}$ & 7,82 & 24,82 & $22.296,00$ \\
\hline$\varnothing$ Wartezeit [s] & 7,82 & 23,17 & $20.045,48$ \\
\hline
\end{tabular}

In den Simulationsergebnissen zeigt sich deutlich, dass die Batchbildung und Priorisierung von Aufträgen das Systemverhalten verändern. Es sei noch einmal darauf verwiesen, dass in allen Experimenten die Ereignisse (Bearbeitungszeit in Servicestation 1 und 2) identischen Verteilungen mit gleichen Mittelwerten und gleichen Varianzen zugrunde liegen.

Diese Beobachtungen sollen die Notwendigkeit aufzeigen, Zufallsprozesse in logistischen Systemen neben Verteilungsfunktion, Mittelwert und Varianz durch weitere Charakteristika zu beschreiben. Ziel ist es, Modelle besser abzubilden, realitätsnähere Simulationsstudien durchzuführen und letztendlich die Systemdimensionierung exakter zu gestalten. Im vorliegenden Fall wird untersucht, inwieweit sich das Ähnlichkeitsmaß der Korrelation bzw. Autokorrelation eignet, um den beschriebenen Herausforderungen gerecht $\mathrm{zu}$ werden. Dazu werden Realdaten auf Autokorrelation untersucht. Im Anschluss daran wird überprüft, ob die ermittelten Maßzahlen genutzt werden können, um eine exaktere Modellierung zu erreichen. Auf Basis einer geeigneten Modellierung ist zukünftig denkbar, Autokorrelation gezielt zu nutzen, um Einfluss auf das Systemverhalten zu nehmen. 


\section{2 (AUTO)KORRELATION ALS ÄHNLICHKEITSMASS}

Statistische Ausführungen und Notationen sind, soweit nicht anders angegeben, an das Werk von [SS01] angelehnt.

Korrelation ist die auf das Intervall $[-1 ;+1]$ skalierte Kovarianz. Die Kovarianz ist eine Kennzahl, die den linearen Zusammenhang zwischen zwei Merkmalen $(X)$ und $(Y)$ beschreibt. Formell berechnet sich die empirische Kovarianz $c$ für $N$ Beobachtungspaare $\left(x_{i}, y_{i}\right)$ durch

$$
\mathrm{c}=\frac{1}{\mathrm{~N}} \sum_{\mathrm{i}=1}^{\mathrm{N}}\left(\mathrm{x}_{\mathrm{i}}-\overline{\mathrm{x}}\right)\left(\mathrm{y}_{\mathrm{i}}-\overline{\mathrm{y}}\right) \mid \overline{\mathrm{x}}=\sum_{\mathrm{i}} \frac{\mathrm{x}_{\mathrm{i}}}{\mathrm{N}} ; \overline{\mathrm{y}}=\sum_{\mathrm{i}} \frac{\mathrm{y}_{\mathrm{i}}}{\mathrm{N}}
$$

Die Normierung erfolgt anhand des Produktes der Standardabweichungen

$$
\mathrm{s}_{\mathrm{x}}=\sqrt{\frac{1}{\mathrm{~N}} \sum_{\mathrm{i}=1}^{\mathrm{N}}\left(\mathrm{x}_{\mathrm{i}}-\overline{\mathrm{x}}\right)^{2}} ; \mathrm{s}_{\mathrm{y}}=\sqrt{\frac{1}{\mathrm{~N}} \sum_{\mathrm{i}=1}^{\mathrm{N}}\left(\mathrm{y}_{\mathrm{i}}-\overline{\mathrm{y}}\right)^{2}},
$$

sodass der Korrelationskoeffizient $r$ nach Bravais-Pearson definiert ist durch

$$
\mathrm{r}=\frac{\mathrm{c}}{\mathrm{s}_{\mathrm{x}} * \mathrm{~s}_{\mathrm{y}}} .
$$

Inhaltlich treffen Korrelationskoeffizienten kausalitätsunabhängige Aussagen über die Richtung und die Stärke des linearen Zusammenhangs der untersuchten Merkmale. Betragsmäßig hohe Korrelationkoeffizienten signalisieren eine starke Abhängigkeit. Bildlich wird bei der Berechnung von $r$ durch die Messwertreihen $x_{i}$ und $y_{i}$ je eine Gerade gelegt, sodass die quadrierten Abstände jeweils minimal werden. Der Winkel zwischen den resultierenden Geraden veranschaulicht grafisch die Korrelation. An dieser Stelle wird deutlich, dass nur die Ähnlichkeit und nicht der betragsmäßige Abstand der Beobachtungspaare durch einen Korrelationskoeffizienten ausgedrückt wird.

Bei der Autokovarianz werden die Beobachtungspaare aus einer einzigen Reihe aber mit zeitlichem Versatz $\tau$ abgeleitet. Die Beobachtungspaare werden also für ein Merkmal $(X)$ mit $\left(x_{t}, x_{t+\tau}\right)$ gebildet. Es ergibt sich

$$
\mathrm{c}_{\tau}=\frac{1}{\mathrm{~N}} \sum_{\mathrm{t}=1}^{\mathrm{N}-\tau}\left(\mathrm{x}_{\mathrm{t}}-\overline{\mathrm{x}}\right)\left(\mathrm{x}_{\mathrm{t}+\tau}-\overline{\mathrm{x}}\right) \mid \tau=0,1, \ldots, \mathrm{N}-1
$$

und analog für die Autokorrelationskoeffizienten $\left(r_{\tau}\right)$

$$
\mathrm{r}_{\tau}=\frac{\sum_{\mathrm{t}=1}^{\mathrm{N}-|\tau|}\left(\mathrm{x}_{\mathrm{t}}-\overline{\mathrm{x}}\right)\left(\mathrm{x}_{\mathrm{t}+|\tau|}-\overline{\mathrm{x}}\right)}{\sum_{\mathrm{t}=1}^{\mathrm{N}}\left(\mathrm{x}_{\mathrm{t}}-\overline{\mathrm{x}}\right)^{2}} .
$$

Der Zeitversatz $\tau$ wird auch als Lag bezeichnet. Inhaltlich geben $r_{\tau}$ Aufschluss darüber, wie ähnlich die Beobachtungsreihe mit sich selber jeweils bei Lag $\tau$ ist. Eine Möglichkeit zur Darstellung von mehreren $r_{\tau}$ einer Zeitreihe zu verschiedenen Lags besteht in sogenannten Autokorrelogrammen (vgl. Abbildung 4).
Wie in Abbildung $4 \mathrm{zu}$ sehen, sind auf der Abszisse Lag-weise die Autokorrelationskoeffizienten abgetragen. Es sei erwähnt, dass $r_{0}$ entsprechend der Definition des Korrelationskoeffizienten immer 1 beträgt. Weiterhin sind gestrichelte Signifikanzlinien eingezeichnet. Sie begrenzen ein Konfidenzintervall und geben an, ob ein $r_{\tau}$ signifikant unterschiedlich zu 0 ist. Tritt ein $r_{\tau}$ aus dem Band heraus, so ist statistisch abgesichert, dass eine Autokorrelation bei Lag $\tau$ besteht. Das Konfidenzband ergibt sich nach folgender Überlegung. Es kann nachgewiesen werden, dass bei hinreichend großem $N$ die Autokorrelationskoeffizienten approximativ unabhängig normalverteilt sind, mit Erwartungswert $\mu$ und Standardabweichung $\sigma$

$$
\mu=-\frac{1}{N} ; \sigma=\sqrt{\frac{1}{N}} .
$$

Für die vorliegende Arbeit wird, soweit nicht anders angegeben, immer ein Signifikanzniveau von 95\% angenommen. Es ergibt sich ein Konfidenzintervall von

$$
\left[-\frac{1}{\mathrm{~N}}-1,96 \cdot \sqrt{\frac{1}{\mathrm{~N}}} ;-\frac{1}{\mathrm{~N}}+1,96 \cdot \sqrt{\frac{1}{\mathrm{~N}}}\right] .
$$

Zur genauen Herleitung siehe z. B. [Ass00]. Weitere Tests auf signifikante Autokorrelation wie z. B. der Ljung-Box-Q-Test, Durbin-Watson-Test oder BreuschGodfrey-AK-Test sind [SS01] bzw. [GMM88] zu entnehmen.

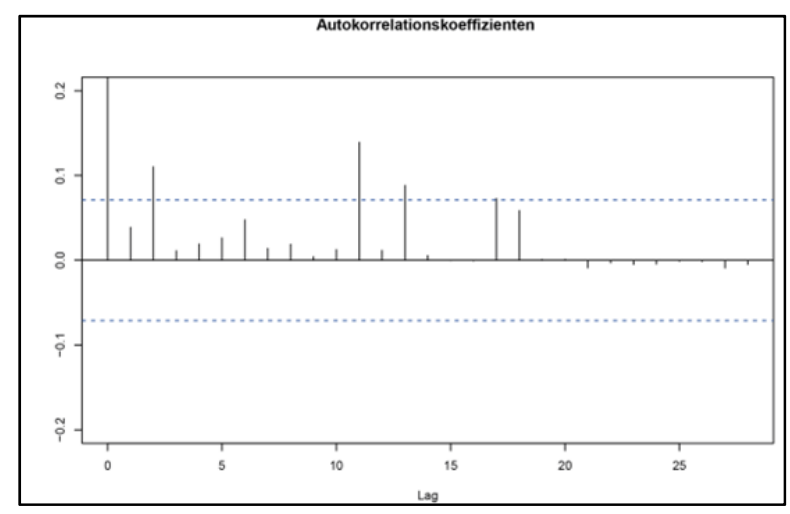

Abbildung 4. Autokorrelogramm einer Beobachtungsreihe mit Signifikanzgrenzen (gestrichelte Linien) Darstellungsintervall Ordinate:[-0,2;0,2]

Weiterhin sollen als Analysewerkzeug die partiellen Autokorrelationskoeffizienten $\left(\alpha_{\tau}\right)$ herangezogen werden. Sie werden aus den $r_{\tau}$ geschätzt und geben den Zusammenhang zwischen Beobachtungswerten $\left(x_{t}, x_{t+\tau}\right)$ an, ohne die indirekte Korrelationen von $x_{u} \mid t<u<t+\tau$ auf $x_{t} \mathrm{zu}$ berücksichtigen. Die Kenntnis über die partiellen Autokorrelationskoeffizienten wird essentiell, wenn ein den Beobachtungwerten zugrundeliegendes Modell geschätzt werden soll. Dargestellt werden die $\alpha_{\tau}$ analog zu den $r_{\tau}$ in einem Korrelogramm (vgl. Abbildung 4). Die Berechnung erfolgt beispielsweise über ein Yule-Walker-Gleichungssystem [SS01; Ach87; Esh03]. 


\section{STAND DER FORSCHUNG}

\subsection{ZUFALLSZAHLENGENERATOREN}

Zufallszahlen sind das Ergebnis eines Zufallsexperimentes. Im vorliegenden Fall werden sie verwendet, um zufällige Ereignisse in Simulationen zu beschreiben.

Folgen von Zufallszahlen werden durch Zufallszahlengeneratoren (englisch: random number generators, RNG) erzeugt. Grundsätzlich wird zwischen echten RNG und Algorithmen unterschieden. Echte RNG leiten ihre Zufallszahlen aus physikalischen Prozessen wie bspw. radioaktiven Zerfallsprozessen oder Signalschwankungen in elektrischen Schaltungen ab. Im wissenschaftlichen und praktischen Umfeld der Logistik spielt diese Art von RNG keine Rolle. Bekannte Simulatoren wie bspw. AutoMod, Plant Simulation, Arena, Simul8, Witness oder auch Programmiersprachen wie Java, C, und Excel/ VBA setzen Algorithmen zur Erzeugung von dann Pseudozufallszahlen ein [Ban00; Lec06; Mad08; Sim12]. Zentrales Anliegen ist immer, identisch verteilte und unabhängige, $d . h$. unkorrelierte Zufallszahlen zu erzeugen [Lec06]. Als Quasistandard haben sich Generatoren etabliert, die auf einer linear rekurrenten Bildungsvorschrift basieren, sogenannte Kongruenzgeneratoren.

Sollen korrelierte Zufallszahlen erzeugt werden, müssen spezielle Verfahren zum Einsatz kommen. Dafür eignet sich z. B. der ARMA-Ansatz nach [BJ70] oder eine Abwandlung in Form eines ARTA-Prozesses nach [CN96]. Eine Distorsions-Methode, die sogenannte TESMethode, wird in [Me191] beschrieben. [LM91] erläutert die Generierung von abhängigen Zufallszahlen mit einem Mini- bzw. Maxification-Ansatz nach [LMT93]. Abschließend sei noch auf die Literatur von [Sk173], [Gla03] und [LMN90] verwiesen. Dort wird beschrieben, wie Copulas, geometrische Brownsche Bewegungen und verstetigte Markov-Ketten eingesetzt werden können, autokorrelierte Zufallszahlen zu generieren.

\subsection{AUTOKORRELATION IN LOGISTISCHEN SYSTEMEN}

Der Einfluss von Autokorrelation auf Bediensysteme ist lange bekannt. Bereits [Run62] unterstellt, dass Ankunftsabstände in Warteschlangensystemen nicht unabhängig Poisson-verteilt sind. Mit dem Übergang von poissonverteilten Zwischenankunftszeiten zu einem MarkovProzess, wird auch die Annahme der Unkorreliertheit aufgeweicht. Der Autor kommt zur Erkenntnis, dass mit steigender Korrelation in einem Single-Server-Queue-Modell mit einer höheren Wahrscheinlichkeit lange Warteschlangen zu beobachten sind.

[LMT93] untersuchen an einem M/M/1-Simulationsmodell die Auswirkung autokorrelierter Zwischenankunftszeiten sowie Bedienzeiten auf die „Performance“ (mittlere Wartezeit der Forderungen) des Modells. Zur Erzeugung entsprechend korrelierter $\mathrm{Zu}$ - fallszahlen nutzen die Autoren die Methoden „TES“ und „Minification“ (vgl. Abschnitt 3.1). Angesichts der Ergebnisse von [LMT93] erscheint es unverständlich, dass nicht bereits vor 20 Jahren angefangen wurde, reale Datensätze von Logistik- und Produktions-Prozessen intensiv auf Autokorrelation zu untersuchen. Es ist dokumentiert, dass insbesondere bei Lag 1 schon moderate Autokorrelation $\mathrm{zu}$ deutlich abweichenden Wartezeiten im Vergleich zu unkorrelierten Zufallszahlen führen. Dies ist unabhängig davon, ob die Ankunfts- oder die Bedienzeiten eine Autokorrelationsstruktur aufweisen. Im Extremfall sind Abweichungen von mehreren $1.000 \%$ zu erwarten.

$\mathrm{Zu}$ ähnlichen Ergebnissen kommen [PDM93]. Sie untersuchen simulativ autokorrelierte Erlang-2- und Poisson-verteilte Ankunftsströme in einem Single-ServerQueue-Modell.

Aktuellere Untersuchungen wie [AM01] wiederholen vor dem Hintergrund der Generierung von autokorrelierten Zufallszahlen den Versuchsaufbau von [LMT93] und kommen zu vergleichbaren Aussagen. Des Weiteren wird simuliert, welchen Effekt korrelierte Zwischenausfallzeiten bei Maschinen auf die mittlere Wartezeit in einem M/G/1-Modell haben. Hier zeigt sich ebenfalls, dass bei zunehmenden positiven Autokorrelationskoeffizienten mit erheblich längeren Wartezeiten zu rechnen ist.

[Nie04] und [Nie07] kann die Ergebnisse von [LMT93] ebenfalls reproduzieren und bestätigen. Es zeigt sich aber, dass die relative Abweichung von Simulationsergebnissen mit korrelierten zu unkorrelierten Zufallszahlen stark abhängig vom verwendeten RNG und dessen Parametrierung ist.

Weitere Literatur über theoretische Betrachtungen von Autokorrelation in Bedienmodellen können den genannten Quellen entnommen werden.

Neben den zitierten Modelluntersuchungen sind keine Studien bekannt, welche die Art, Ursachen und Auswirkungen von Autokorrelation in der Logistik beschreiben. Dies deckt sich mit der Aussage in Abschnitt 3.1, wonach in kommerziellen (Simulations-)Softwarepaketen standardmäßig linear rekursive RNG eingesetzt werden. Der Anspruch hierbei, möglichst eine hohe Unabhängigkeit der Zufallszahlen zu erreichen, führt im Umkehrschluss dazu, dass Autokorrelation abseits der Wissenschaft keine Rolle spielt. Dies wird durch eine Aussage von Barry L. Nelson ${ }^{1}$ in jeglicher Hinsicht bestätigt. Dieser lässt sich auf Rückfrage am 26.02.2009 zitieren, dass sowohl bei Softwareentwicklern als auch bei Anwendern

\footnotetext{
${ }^{1}$ Department of Industrial Engineering \& Management Sciences, McCormick School of Engineering and Applied Science, Northwestern University
} 
kein Bedarf besteht, von der Unabhängigkeitsforderung an die Zufallszahlen abzuweichen, obschon das Problem teilweise bekannt ist, aber unterschätzt wird. $\mathrm{Zu}$ ähnlicher Einschätzung kommen auch [YAG05]. Einen weiteren Grund für das Festhalten an unkorrelierten Zufallszahlen sehen [LMT93] in der statistisch einfacheren Handhabbarkeit.

\section{Untersuchung Logistischer Prozesse auf AUTOKORRELATION}

Der folgende Abschnitt behandelt die Untersuchung von Logistik- und Produktionsprozessen auf Autokorrelation.

\subsection{EXPERIMENTAUFBAU}

Alle zu untersuchenden Datensätze (vgl. Abschnitt 4.2) enthalten eine Folge von Forderungen/ Aufträgen mit jeweils einem Zeitstempel des Entstehens. Aus Modellierungssicht sind dies die Eintrittszeitpunkte in die Warteschlange vor einer Bedienstation. Aus den Zeitstempeln werden Zwischenankunftszeiten berechnet. Sämtliche Zeitreihen werden vorher um Stillstandszeiten (Feierabend, Betriebsruhe, Sonn-, Feiertage) bereinigt, sodass ein nahtloser Übergang zwischen den Aufträgen stattfindet. Inhaltlich werden die Daten nicht weiter aufgearbeitet, also bspw. weder vorselektiert noch vermeintliche Ausreißer entfernt.

Aus den Datensätzen werden jeweils logisch/ ablauftechnisch zusammenhängende Teildatensätze gebildet und untersucht. Ein Subdatensatz (SubD) enthält ungefähr 1.000 bis 6.000 Zwischenankunftszeiten.

Für alle Teildatensätze werden die Autokorrelationskoeffizienten und die partiellen Autokorrelationskoeffizienten bis ca. zum Lag $\tau \mid \tau \approx 10 \log _{10}(\mathrm{~N})$ berechnet [DH08]. Zuvor werden alle Zeitreihen mittels des Augmented-Dickey-Fuller-Tests [DF79] auf Trends untersucht. Im Falle einer trendbehafteten Zeitreihe wären Autokorrelationskoeffizienten als Indikator für Abhängigkeit weniger gut geeignet [SS01]. Für jeden Subdatensatz werden außerdem für die Menge der $r_{\tau}$ und $\alpha_{\tau}$ die Extremwerte und die Absolutmittelwerte notiert. ${ }^{2}$

In einem nächsten Schritt werden die Subdatensätze jeweils einem G/D/1- und einem G/M/1-Simulationsmodell zugeführt. Der Ankunftsprozess wird dabei durch die Datensätze beschrieben.

Parallel wird jeder Teildatensatz in 100 weiteren Experimenten als G/D/1-Modell simuliert, wobei mit jedem

\footnotetext{
${ }^{2}$ Koeffizienten zum Lag 0 werden nicht beachtet. Diese haben immer den Wert 1 (vgl. Abschnitt 2).
}

Simulationslauf die Zwischenankunftszeiten des Datensatzes permutiert werden. Da lediglich permutiert wird, ist sichergestellt, dass die Verteilung und deren Mittelwert sowie Varianz unverändert bleiben. Durch das Umordnen der Zwischenankunftszeiten wird nur deren gegebenenfalls vorhandene Autokorrelationsstruktur zerstört. Auf diese Weise kann für jeden Teildatensatz eine unkorrelierte Referenzstatistik erstellt werden.

In einer zusätzlichen Studie wird pro Subdatensatz die mittlere Zwischenankunftszeit $\mu_{i}$ errechnet und ein $\operatorname{Exp}\left(\mu_{i}\right)$-verteilter Ankunftsstrom in einem M/D/1-Modell 100-mal simuliert. Dieses Experiment entspricht dem in vorangegangen Kapiteln beschriebenen Stand der Technik und gründet auf der Annahme, dass Ankunftsabstände in realen Systemen meist als exponentialverteilt beschrieben werden können [LK00].

Die Bedienzeit der Servicestation wird stets so gewählt, dass sich in Abhängigkeit der Zwischenankunftszeiten eine theoretische Auslastung von 90\% ergibt. Als Ergebnis der Simulationen werden die maximale und mittlere Warteschlangenlänge sowie das 0,75- und 0,90Quantil ${ }^{3}$ protokolliert.

Die Kennzahlen der Autokorrelation und die Simulationsergebnisse werden in Abschnitt 4.3 tabellarisch und grafisch gegenübergestellt.

\subsection{DATENSÄTZE}

Analysiert werden aus der Praxis bereitgestellte Datensätze. In Summe können 5 Datensätze mit insgesamt 52 Teildatensätzen auf Autokorrelation untersucht werden.

Datensatz [A] entstammt einem kleinen Produktionssystem. Sämtliche Transporteinheiten wurden an diversen Punkten zeitlich erfasst. Der Untersuchungszeitraum erstreckt sich auf 12 Werktage. Etwa 50 Datenbankfelder (Tracking-ID einer Palette an einer Lichtschranke) wurden leer vorgefunden, konnten aber mit hoher Konfidenz korrekt rekonstruiert werden. Ein Subdatensatz wird gebildet aus den Zwischenankunftszeiten von Paletten vor einer spezifischen Servicestation des Systems.

In Datensatz [B] sind Entstehungszeitpunkte von Transportaufträgen erfasst. Ein Stapler fungiert in diesem Fall als Servicestation. Ein Subdatensatz wird gebildet aus den Zwischenankunftszeiten von Aufträgen für eine spezifische Quelle-Senke-Relation („Tour“). Es liegen die Transportaufträge für ca. 30 Tage vor.

\footnotetext{
${ }^{3}$ Quantile werden mittels P2-Alogrithmus nach [JC85] geschätzt.
} 
Datensatz [C] erfasst Auslageraufträge eines Automobilherstellers für 10 Werktage. Die Auswertung erfolgt tageweise (entspricht Subdatensatz).

Im Datensatz [D] sind Auslageraufträge eines Logistikzentrums für 8 Werktage dokumentiert. Subdatensätze werden wiederum tageweise abgeleitet.

In Datensatz [E] wurden die Einlastungszeitpunkte von Produktionslosen aufgezeichnet. Inwieweit der Einlastung bereits eine Reihenfolgeplanung zu Grunde liegt, wurde aus Gründen der Verschwiegenheit nicht übermittelt. Der Erfassungszeitraum erstreckt sich über ca. ein Jahr. Subdatensätze werden in Abhängigkeit verschiedener Servicestationen gebildet.

\subsection{ERgEBNISSE}

Nachfolgend in den Abbildungen 5 bis 10 sind Autokorrelogramme einzelner ausgesuchter Subdatensätze dargestellt. Die Grafiken sind repräsentativ für alle Zeitreihen eines Datensatzes zu verstehen. Um einen intuitiven Zugang und eine Vergleichbarkeit zwischen den Grafiken zu ermöglichen, ist auf der Ordinate jeweils nur das Intervall $[-0,2 ; 0,4]$ dargestellt.

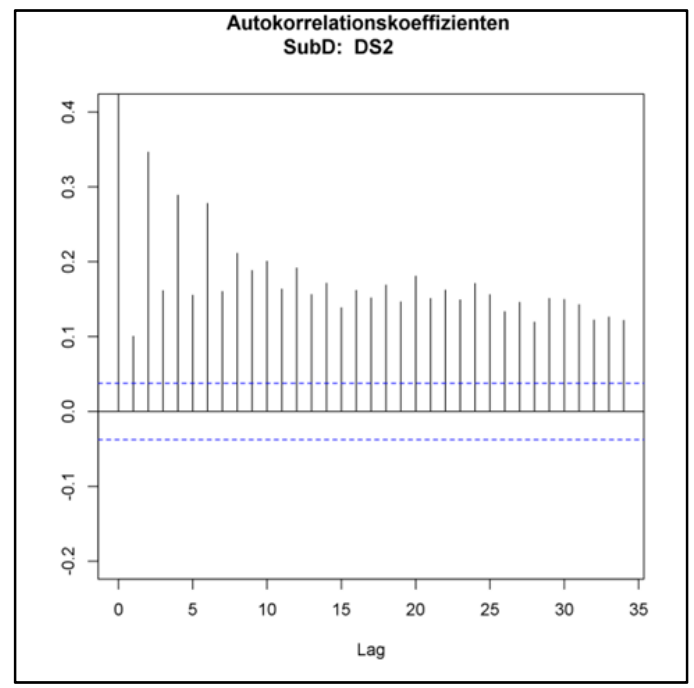

Abbildung 5. Repräsentat. Autokorrelogramm Datensatz [A]

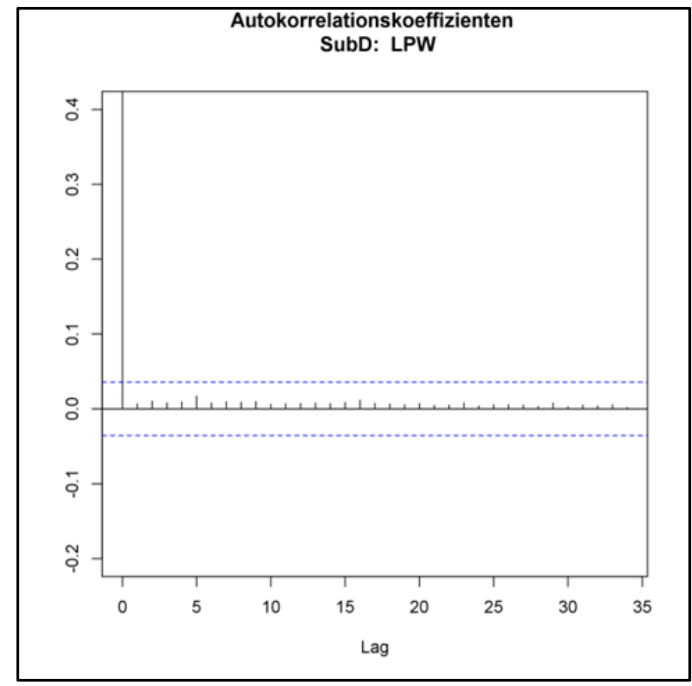

Abbildung 6. Repräsentat. Autokorrelogramm Datensatz [A]

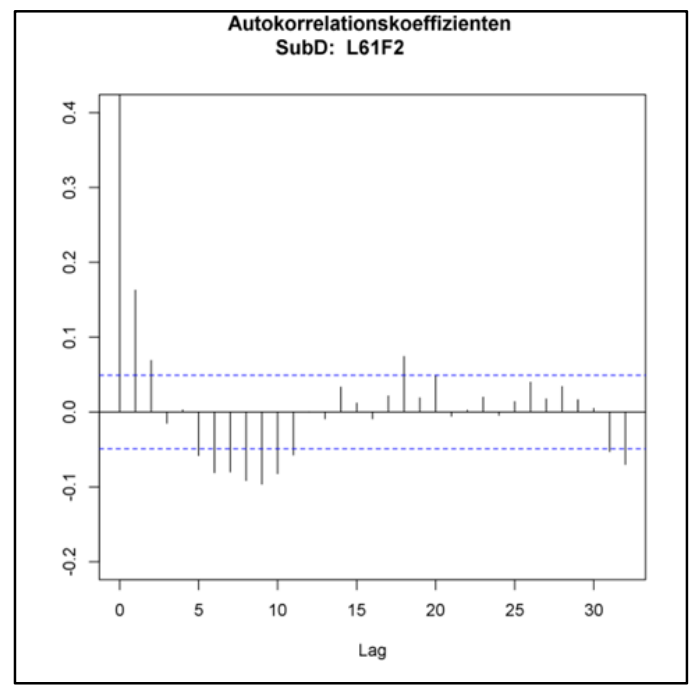

Abbildung 7. Repräsentat. Autokorrelogramm Datensatz [B]

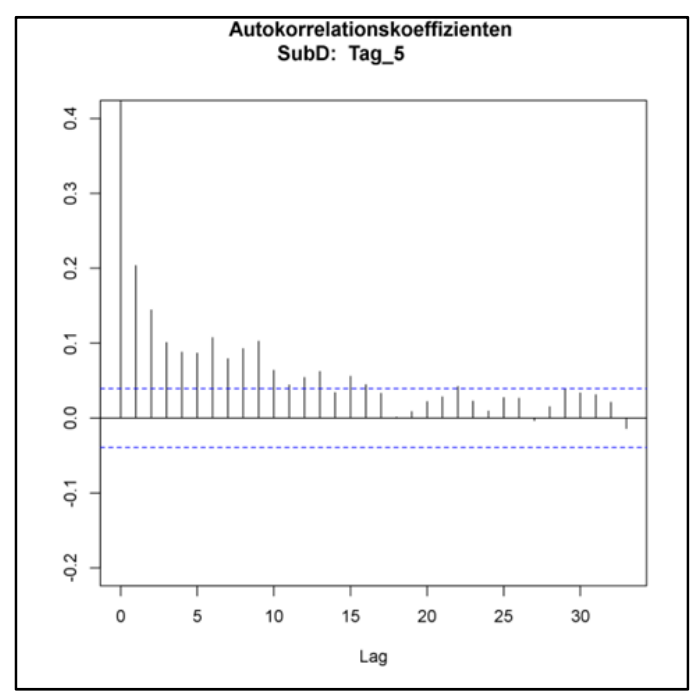

Abbildung 8. Repräsentat. Autokorrelogramm Datensatz [C] 


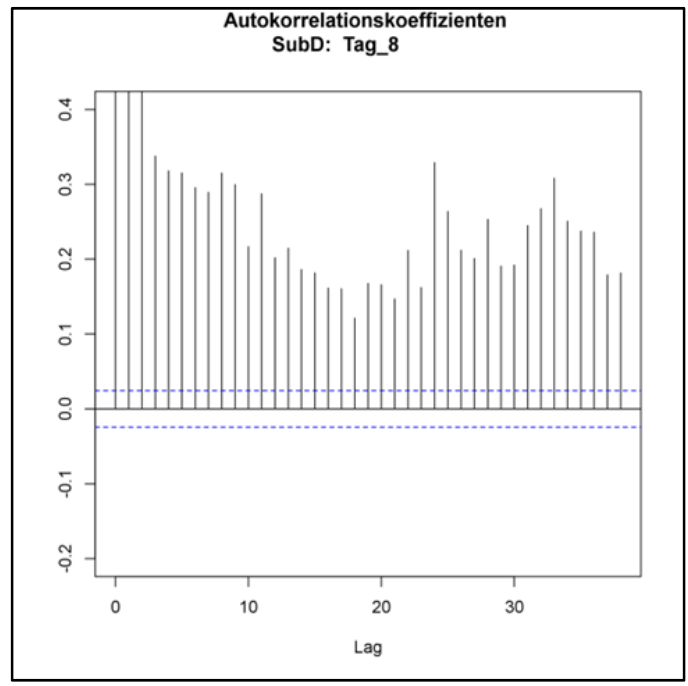

Abbildung 9. Repräsentat. Autokorrelogramm Datensatz [D]

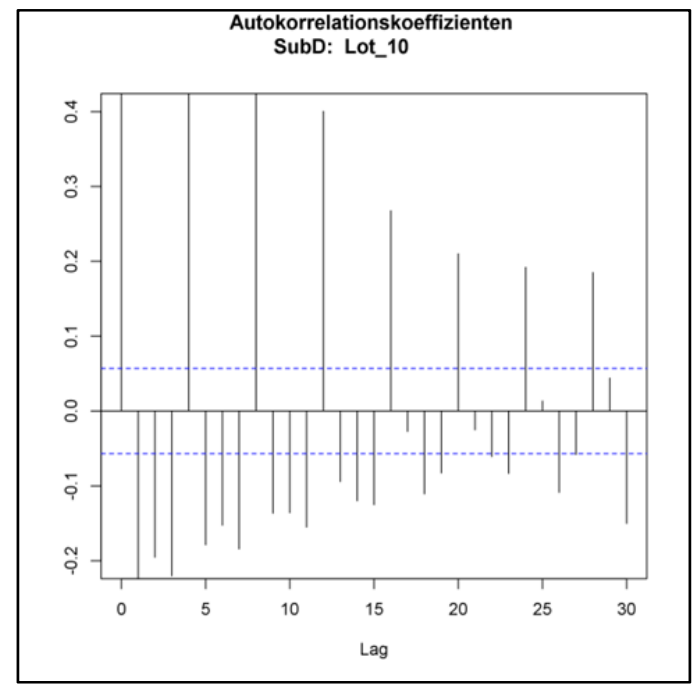

Abbildung 10. Repräsentat. Autokorrelogramm Datensatz [E]

In Tabelle 2 sind Simulationsergebnisse den $r_{\tau}$ gegenübergestellt. Grundlage ist ein G/D/1-Modell. ${ }^{4}$ Dargestellt sind für die einzelnen Teildatensätze die ersten neun $r_{\tau}$ und die mittlere Warteschlangenlänge. ${ }^{5}$ Es werden für jeden Subdatensätze folgende drei Fälle unterschieden (vgl. auch Abschnitt 4.1): Simulation der Realdaten, der permutierten Daten und der mittels Exponentialverteilung modellierten Daten. Für die modellierten und permutier-

\footnotetext{
${ }^{4}$ Die Ergebnisse der Simulationen mit einem G/M/1-Modell werden der Übersicht halber nicht mit dargestellt, ähneln aber denen mit G/D/1-Modell stark. Die Ergebnisse können auf Anfrage bereitgesellt werden.

${ }^{5}$ Weitere eingangs erwähnte Kennzahlen werden der Übersicht halber nicht explizit dargestellt. Inhaltlich ergeben sich keine grundlegend verschiedenen Aussagen zu den vorgestellten Ergebnissen. Alle Kennzahlen können auf Anfrage bereitgestellt werden.
}

ten Daten werden die durchschnittliche mittlere Warteschlangenlänge (Ø) und deren Standardabweichung (SD) aus den 100 Simulationsläufen angegeben.

Die Abbildungen 11 bis 15 bereiten einzelne Ergebnisse aus Tabelle 2 grafisch auf. Es wird auf der Ordinate die mittlere Warteschlangenlänge über den Extremwert der $r_{\tau}$ der einzelnen Subdatensätze abgetragen. ${ }^{5}$ Die Werte der Subdatensätze sind mit einem ,"“ schwarz dargestellt. Jeder dieser Punkte ist durch eine Linie mit einem grauen „“ verbunden. Der graue Punkt repräsentiert die Werte der zugehörigen Referenzstatistik ( $\varnothing$ Permutiert) eines Teildatensatzes, wobei der maximale $r_{\tau}$ mit 0 angegeben wird. ${ }^{6}$ Ist ein Datenpunkt mit einem ,,$“$ veranschaulicht, bedeutet dies, dass mindestens ein Autokorrelationskoeffizient des Subdatensatzes signifikant ist. Jeder Datenpunkt erhält einen Bezeichner. Über diesen wird auf das entsprechende Autokorrelogramm referenziert. Den Grafiken kann unter anderem der Einfluss der stärksten Autokorrelation auf die mittlere Warteschlangenlänge entnommen werden. Gleichzeitig kann anhand der Steigungen der Geraden abgeschätzt werden, ob die unkorrelierten Pendants im Schnitt zu verschiedenen Ergebnissen führen. Das Verhältnis von , $\boldsymbol{\otimes} “ \mathrm{zu},, “$ “ verdeutlicht den Anteil von autokorrelierten $\mathrm{zu}$ nicht autokorrelierten Datensätzen.

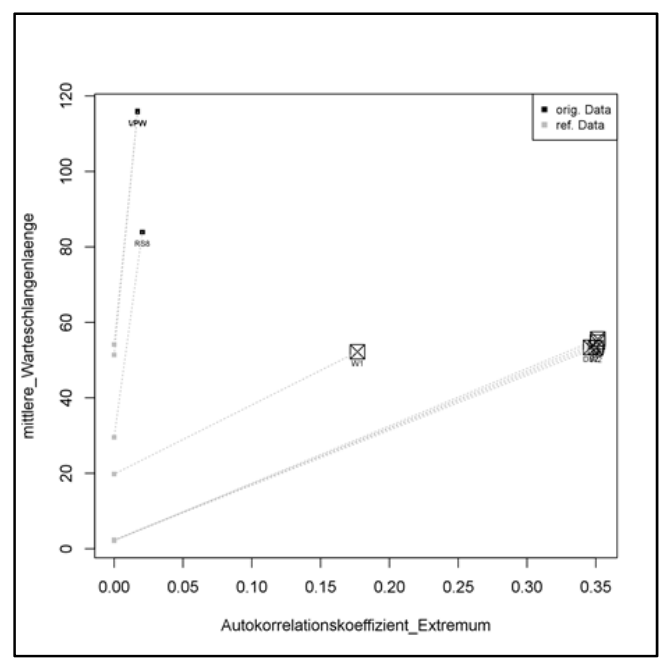

Abbildung 11. Mittlere Warteschlangenläge über maximalen absoluten Autokorrelationskoeffizienten Datensatz [A]

\footnotetext{
${ }^{6}$ Rein statistisch ist $\max \left(\left|\mathbf{r}_{\tau}\right|\right)=0$ nahezu ausgeschlossen. Es soll aber zum Ausdruck kommen, dass der Referenzstatistik Daten ohne signifikante Autokorrelationsstruktur zugrunde liegen.
} 
Tabelle 2. Zusammenfassung Ergebnisse - fett: Autokorrelationskoeffizient $r_{\tau}$ signifikant; grau: Simulation der realen Daten signifikant unterschiedlich zu Simulation der permutierten Daten [Durchschnitt (Ø) + 3·Standardabweichung (SD)]

\begin{tabular}{|c|c|c|c|c|c|c|c|c|c|c|c|c|c|c|c|}
\hline \multirow{3}{*}{$\begin{array}{l}\text { Daten } \\
\text { satz }\end{array}$} & \multirow{3}{*}{$\begin{array}{l}\text { Teildaten } \\
\text { satz }\end{array}$} & \multirow{3}{*}{\multicolumn{9}{|c|}{$\begin{array}{c}\text { Autokorrelationskoeffizient (Lag } 1 \text { bis } 9 \text { ) } \\
\text { der realen Daten }\end{array}$}} & \multicolumn{5}{|c|}{ Simulierte mittlere Warteschlangenlänge } \\
\hline & & & & & & & & & & & \multirow{2}{*}{$\begin{array}{l}\text { Reale } \\
\text { Daten }\end{array}$} & \multicolumn{2}{|c|}{ Permutiert } & \multicolumn{2}{|c|}{ Modelliert } \\
\hline & & & & & & & & & & & & & SD & & SD \\
\hline \multirow{8}{*}[\mathrm{A}]{} & DS1 & 0,14 & 0,35 & 0,22 & 0,32 & $\overline{0,24}$ & 0,29 & $\overline{0,23}$ & 0,25 & 0,21 & 55.59 & 2.15 & 0.29 & 3.99 & 0.96 \\
\hline & DS2 & 0,10 & 0,35 & 0,16 & 0,29 & 0,16 & 0,28 & 0,16 & 0,21 & 0,19 & 53.37 & 2.38 & 0.40 & 3.99 & 1.46 \\
\hline & HS & 0,13 & 0,35 & 0,22 & 0,31 & 0,23 & 0,29 & 0,22 & 0,24 & 0,22 & 54.78 & 2.15 & 0.34 & 4.09 & 1.05 \\
\hline & LPW & 0.01 & 0.01 & 0.01 & 0.01 & 0.02 & 0.01 & 0.01 & 0.01 & 0.01 & 115.97 & 51.39 & 13.48 & 3.87 & 0.98 \\
\hline & RS8 & 0.02 & 0.00 & -0.01 & -0.01 & -0.01 & -0.01 & 0.00 & 0.02 & 0.00 & 83.95 & 29.54 & 10.29 & 4.05 & 0.96 \\
\hline & VPW & 0.01 & 0.01 & 0.01 & 0.01 & 0.02 & 0.01 & 0.01 & 0.01 & $0.01 \|$ & 115.78 & 54.14 & 14.47 & 4.00 & 0.96 \\
\hline & W1 & 0.04 & 0,14 & 0.01 & 0.03 & 0.03 & 0.06 & 0.02 & 0.03 & 0.01 & 52.19 & 19.79 & 5.77 & & 1.97 \\
\hline & w2 & 0,11 & 0,35 & 0,18 & 0,30 & 0,16 & 0,29 & 0,17 & 0,21 & 0,20 & 53.26 & 2.24 & 0.32 & 9 & 1.08 \\
\hline \multirow{20}{*}{ [B] } & D11A & 0,07 & 0.02 & 0,04 & 0.02 & -0.01 & -0.02 & $-0,03$ & -0.02 & $-0,03$ & \begin{tabular}{l|}
30.62 \\
\end{tabular} & 41.98 & 11.76 & 19 & 0.80 \\
\hline & D11B1 & 0.03 & -0.01 & -0.03 & -0.04 & -0.03 & 0.01 & -0.02 & -0.03 & -0.01 & 14.11 & 18.90 & 5.42 & 4 & 1.57 \\
\hline & D11D & 0.02 & -0.02 & 0.01 & -0.03 & 0.00 & 0.00 & -0.02 & -0.03 & -0.03 & 9.92 & 17.31 & 4.66 & .87 & 1.00 \\
\hline & D 21C2 & 0,13 & 0,06 & 0.01 & -0.01 & -0.01 & -0.02 & -0.04 & $-0,06$ & -0.03 & 30.34 & 19.22 & 4.75 & 4.00 & 1.26 \\
\hline & E31A1 & 0,15 & 0.02 & -0.01 & -0.04 & $-0,06$ & $-0,06$ & -0.05 & $-0,06$ & $-0.01 \|$ & 14.95 & 18.82 & 5.62 & 86 & 1.36 \\
\hline & ЕТНH & 0,15 & 0.03 & 0,06 & 0.01 & 0.00 & 0.02 & 0.00 & 0.01 & 0.00 & 46.38 & 33.55 & 10.12 & 8 & 1.07 \\
\hline & H11S2 & 0.05 & -0.02 & 0.06 & 0.05 & 0.00 & 0.09 & 0.03 & -0.02 & 0.06 & 10.09 & 6.59 & 2.13 & 4 & 1.89 \\
\hline & L31S3 & 0.06 & -0.03 & -0.05 & -0.03 & -0.04 & -0.04 & 0.01 & 0.03 & -0.03 & 6.01 & & 3.42 & & 1.72 \\
\hline & L31S4 & 0,08 & 0.00 & 0.00 & -0.06 & -0.06 & 0.01 & 0.02 & 0.01 & 0.03 & 14.07 & & & & 1.60 \\
\hline & L31kI.E & 0,16 & 0,06 & -0.01 & $-0,05$ & $-0,05$ & $-0,06$ & $-0,06$ & -0.01 & $-0,05$ & 16.42 & 16.95 & 4.37 & 4 & 1.31 \\
\hline & L41F1 & 0,06 & 0,06 & 0.02 & 0.00 & 0.01 & 0.00 & 0.00 & -0.02 & -0.02 & 20.29 & 30.68 & 8.17 & 9 & 1.24 \\
\hline & L51F1 & 0,05 & 0.00 & 0.01 & -0.01 & -0.03 & 0.02 & -0.02 & -0.03 & $-0,05$ & 15.14 & 23.50 & 6.65 & 3 & 1.30 \\
\hline & L61F2 & 0,16 & 0,07 & -0.01 & 0.00 & $-0,06$ & $-0,08$ & $-0,08$ & $-0,09$ & $-0,10$ & 11.29 & 17.61 & 4.73 & 4 & 1.22 \\
\hline & L91F2 & 0,11 & 0.00 & -0.03 & -0.03 & -0.04 & -0.02 & -0.01 & -0.03 & -0.03 & 40.75 & 21.19 & 5.64 & 3 & 1.13 \\
\hline & La ge r15 & 0,05 & 0.01 & 0.02 & -0.01 & -0.01 & 0.02 & 0.00 & -0.03 & $-0.01 \|$ & 15.57 & 3 & 4.07 & 4 & 1.56 \\
\hline & M11 & 0.01 & 0.00 & 0.01 & 0.00 & 0.00 & 0.02 & -0.01 & -0.02 & $0.01 \|$ & 31.67 & 19 & 8.95 & 8 & 1.56 \\
\hline & REST & 0,05 & 0.01 & 0.02 & 0.01 & 0,04 & 0.03 & -0.01 & 0,04 & 0,09 & 65.56 & 35.24 & 9.76 & 0 & 1.18 \\
\hline & S11.1 & 0,23 & 0,15 & 0,12 & 0,06 & 0.05 & 0.01 & -0.01 & $-0,06$ & -0.04 & 22.60 & 14.96 & 5.11 & 3.92 & 1.56 \\
\hline & in & 0,07 & 0,05 & 0,03 & 0.01 & 0,03 & 0.00 & 0.00 & 0.00 & $0.01 \|$ & 29.42 & 26.57 & 6.08 & 4. & 0.99 \\
\hline & Z21 & 0,16 & 0,12 & 0.07 & 0.07 & -0.03 & 0.04 & -0.03 & 0.04 & 0.04 & 12.72 & 7.21 & $1 . \varepsilon$ & 58 & 1.79 \\
\hline \multirow{10}{*}[C]{} & 1 & 0,14 & 0,16 & 0,28 & 0,11 & 0,10 & 0,15 & 0,06 & 0,07 & 0,09 & 171.00 & 15.85 & 4.22 & 4 & 1.25 \\
\hline & Tag_2 & 0,06 & 0,11 & 0,10 & 0,10 & 0,07 & 0,08 & 0.04 & 0.03 & 0,06 & 115.33 & 14.25 & 4.29 & 6 & 1.05 \\
\hline & Tag_3 & 0,13 & 0,12 & 0,10 & 0,12 & 0,12 & 0,10 & 0,06 & 0,06 & 0,07 & 70.93 & 11.28 & 3.25 & 5 & 1.48 \\
\hline & Tag_4 & 0,12 & 0,10 & 0,05 & 0,10 & 0.04 & 0,07 & 0.04 & 0.04 & 0,05 & 77.74 & 13.61 & 3.84 & 3.92 & 1.02 \\
\hline & Tag_5 & 0,20 & 0,14 & 0,10 & 0,09 & 0,09 & 0,11 & 0,08 & 0,09 & 0,10 & 74.49 & 11.86 & 3.01 & 3.99 & 1.14 \\
\hline & Tag_6 & 0,23 & 0,16 & 0,19 & 0,10 & 0,07 & 0,04 & 0,06 & 0,10 & 0,04 & 221.90 & 16.11 & 3.57 & 3.91 & 1.15 \\
\hline & Tag_7 & 0,12 & 0,08 & 0,10 & 0,05 & 0,12 & 0.04 & 0,06 & 0.04 & 0.03 & 54.17 & 12.54 & 3.19 & 4.07 & 1.22 \\
\hline & Tag_8 & 0,13 & 0,13 & 0,12 & 0,12 & 0,07 & 0,05 & 0,05 & 0,05 & 0.04 & 45.92 & 13.79 & 4.03 & 4.03 & 1.23 \\
\hline & Tag_9 & 0,12 & 0,14 & 0,09 & 0,08 & 0,06 & 0,08 & 0,07 & 0,09 & 0,07 & 65.47 & 13.31 & 3.22 & 3.89 & 1.12 \\
\hline & Tag_10 & 0,13 & 0,12 & 0,06 & 0,07 & 0,09 & 0,08 & 0,05 & 0,04 & 0,04 & 89.30 & 15.11 & 4.17 & 4.15 & 1.13 \\
\hline \multirow{8}{*}{ [D] } & Tag_1 & 0,45 & 0,53 & 0,43 & 0,45 & 0,44 & 0,39 & 0,32 & 0,36 & 0,25 & 466.21 & 27.61 & 6.80 & 5 & 0.69 \\
\hline & Tag_2 & 0,30 & 0,40 & 0,27 & 0,49 & 0,29 & 0,28 & 0,28 & 0,36 & $0,34 \|$ & 432.49 & 30.25 & 7.15 & 3.98 & 0.75 \\
\hline & Tag_3 & 0,36 & 0,50 & 0,30 & 0,33 & 0,32 & 0,19 & 0,19 & 0,16 & $0,21 \|$ & 425.25 & 32.63 & 6.89 & 4.02 & 0.76 \\
\hline & Tag_4 & 0,49 & 0,51 & 0,54 & 0,39 & 0,37 & 0,34 & 0,35 & 0,39 & 0,32 & 456.15 & 28.91 & 6.21 & 3.95 & 0.81 \\
\hline & Tag_5 & 0,58 & 0,45 & 0,39 & 0,36 & 0,30 & 0,21 & 0,17 & 0,22 & $0,24 \|$ & 325.62 & 29.71 & 8.89 & 3.89 & 0.67 \\
\hline & Tag_6 & 0,45 & 0,41 & 0,35 & 0,35 & 0,32 & 0,25 & 0,28 & 0,21 & 0,21 & 398.85 & 16.27 & 4.03 & 4.04 & 0.82 \\
\hline & Tag_7 & 0,46 & 0,47 & 0,38 & 0,42 & 0,35 & 0,40 & 0,39 & 0,32 & 0,31 & 526.35 & 25.65 & 5.70 & 4.09 & 0.88 \\
\hline & Tag_8 & 0,46 & 0,42 & 0,34 & 0,32 & 0,32 & 0,30 & 0,29 & 0,32 & 0,30 & 518.39 & 23.15 & 5.58 & 4.01 & 0.77 \\
\hline \multirow{6}{*}[E]{} & Lot_11 & $-0,17$ & $-0,17$ & $-0,17$ & 0,52 & $-0,16$ & $-0,12$ & $-0,13$ & 0,45 & $-0,14$ & 51.74 & 16.37 & 4.60 & 3.98 & 0.93 \\
\hline & Lot_16 & $-0,22$ & $-0,19$ & $-0,18$ & 0,52 & $-0,19$ & $-0,12$ & $-0,12$ & 0,39 & $-0,16$ & 3.94 & 12.69 & 3.60 & 3.87 & 1.35 \\
\hline & Lot_10 & $-0,23$ & $-0,20$ & $-0,22$ & 0,61 & $-0,18$ & $-0,15$ & $-0,18$ & 0,46 & $-0,14$ & 6.86 & 12.35 & 3.49 & 4.12 & 1.44 \\
\hline & Lot_13 & $-0,17$ & $-0,19$ & $-0,19$ & 0,36 & $-0,07$ & $-0,12$ & $-0,13$ & 0,22 & -0.02 & 5.12 & 13.52 & 4.52 & 3.67 & 1.22 \\
\hline & Lot_97 & $-0,16$ & $-0,09$ & $-0,13$ & 0,22 & $-0,10$ & -0.03 & $-0,08$ & 0.06 & -0.04 & 5.45 & 10.37 & 2.65 & 3.73 & 1.21 \\
\hline & Lot_99 & $-0,16$ & $-0,17$ & $-0,17$ & 0,33 & $-0,09$ & $-0,11$ & $-0,09$ & 0,25 & $-0,04$ & 33.95 & 17.28 & 4.01 & 4.05 & 0.97 \\
\hline
\end{tabular}




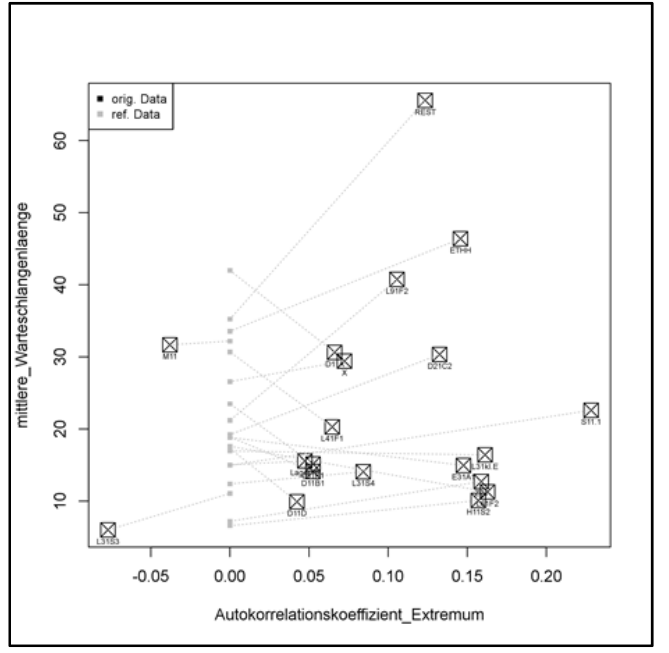

Abbildung 12. Mittlere Warteschlangenläge über maximalen absoluten Autokorrelationskoeffizienten Datensatz [B]

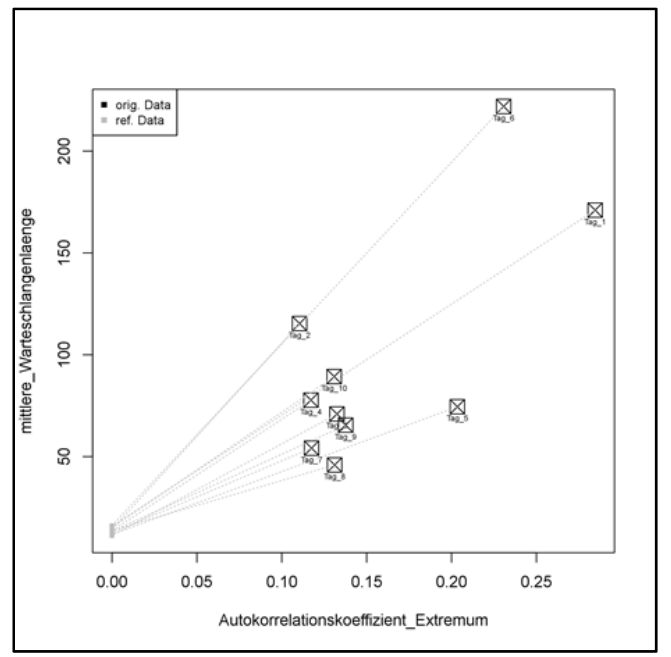

Abbildung 13. Mittlere Warteschlangenläge über maximalen absoluten Autokorrelationskoeffizienten Datensatz [C]

\section{Auswertung}

Im Folgenden werden die erzielten Ergebnisse bewertet und insbesondere bezüglich des Vorkommens von Autokorrelation in den Realdaten betrachtet.

Laut Teststatistik des Augmented-Dickey-FullerTests, weisen fast alle Teildatensätze mit einem Signifikanzniveau von $99 \%$ keinen Trend auf. Ausnahme bilden die Subdatensätze Tag_2, Tag_3 und Tag_5 aus Datensatz [D]. Hier ist die Interpretation der Autokorrelationskoeffizienten nicht eindeutig (vgl. Kapitel 2 und 4). ${ }^{7}$

\footnotetext{
${ }^{7}$ Im weiteren Verlauf des Kapitels erfolgt die Auswertung teils unabhängig von den Ergebnissen der Augmented-DickeyFuller-Teststatistiken.
}

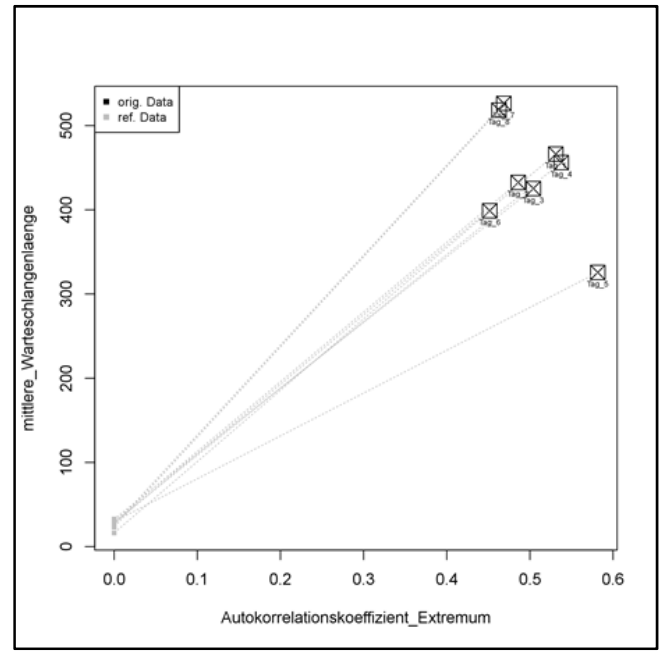

Abbildung 14. Mittlere Warteschlangenläge über maximalen absoluten Autokorrelationskoeffizienten Datensatz [D]

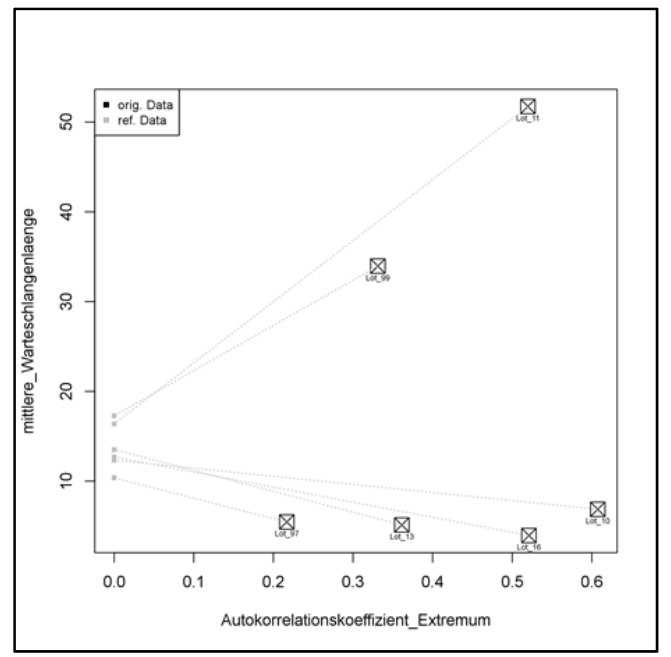

Abbildung 15. Mittlere Warteschlangenläge über maximalen absoluten Autokorrelationskoeffizienten Datensatz [E]

In insgesamt 49 von 52 Teildatensätzen kann mindestens ein signifikanter $r_{\tau}$ gefunden werden. Dies entspricht einer Quote von 94\%. Einzig Datensatz [A] enthält 3 Subdatensätze, die keine signifikante Autokorrelationsstruktur aufweisen.

Global über alle Datensätze sind völlig unterschiedliche Autokorrelationsmuster zu erkennen. In Datensatz [A] lassen sich signifikant nur positive $r_{\tau}$ nachweisen. Die Werte in [B] folgen dagegen einer wellenförmigen Funktion auf. Oft wird das Signifikanzband hier nur geringfügig verlassen. In [C] und [D] können unregelmäßige Autokorrelationsstrukturen mit teils stark positiven $r_{\tau}$ beobachtet werden. Bemerkenswert ist der Verlauf der Korrelogramme in [E]. Es bilden sich wiederkehrende Muster aus jeweils drei negativen gefolgt von einem positiven $r_{\tau}$. Dies ist vermutlich auf eine stark ausgeprägte Maschinenbelegungsplanung zurückzuführen. 
Die betragsmäßig größten Autokorrelationskoeffizienten, auch bei großen Lags, treten in Datensatz [D] auf. Dazu passt das Ergebnis der zugehörigen Simulation. Die längsten mittleren Warteschlangen sind bei den entsprechenden Teildatensätzen zu beobachten. Für [E] sind, mit Ausnahmen, die kürzesten Warteschlangenlängen zu verzeichnen. Erstaunlich an dieser Stelle ist die Tatsache, dass sich für [A] die längsten Warteschlangen bei Subdatensätzen ohne signifikante Autokorrelationsstruktur einstellen.

Die durchschnittliche mittlere Warteschlangenlänge bei Simulation permutierter Subdatensätze führt zu uneinheitlichen Ergebnissen. Einzig bei [D] werden konstant vergleichsweise große Warteschlangen beobachtet. Erwähnenswert in diesem Zusammenhang sind wiederum die Ergebnisse von [A]. Jene Teildatensätze, die originär keine Autokorrelation aufweisen, erzielen auch im permutierten Fall die durchschnittlich längsten Warteschlangen.

Werden die Spalten „Reale Daten“ und „Permutiert" aus Tabelle 2 verglichen zeigt sich zudem, dass in ca. $75 \%$ der Fälle die Originaldaten eine längere Warteschlangenlänge verursachen. In den Grafiken 11 bis 15 wird dies anhand der positiven Steigung der Geraden deutlich. Bei [E] kehrt sich der Effekt aufgrund der negativen $r_{\tau}$ teils um. Wird [B] betrachtet, ist das Ergebnis uneinheitlich. Eine Erklärung wäre, dass die Autokorrelationsstrukturen in den Daten von [B] teilweise nur „moderat“" ausfallen.

Bei Datensätzen mit ausgeprägten $r_{\tau}$ zeigt sich, dass die mittlere Warteschlangenlänge für die Realdaten häufig außerhalb des $3 \sigma$-Bereichs ${ }^{8}$ der Referenzstatistik liegt (grau hinterlegt). Dies kann als deutliches Anzeichen gesehen werden, dass die Warteschlangenlängen entsprechender Datensätze nicht zufällig hohe Werte aufweisen, sondern dies auf Autokorrelation zurückzuführen ist.

In Tabelle 2 wird außerdem deutlich, dass nur in wenigen Fällen die modellierten Daten das System valide abbilden würden. Wie in den bisherigen theoretischen Betrachtungen bereits aufgezeigt (vgl. Kapitel 3.2), wird auch bei den hier untersuchten Realdaten deutlich, dass unkorrelierte Zufallszahlen mit einer üblichen Exponentialverteilung das System lediglich unzureichend abbilden. Es ergeben sich teilweise Abweichungen von mehreren tausend Prozent.

Die in den Absätzen zuvor dargelegten Erkenntnisse beziehen sich auf ein G/D/1-Modell. Im Kern gleiche Erkenntnisse ergeben sich bei Grundlage eines G/M/1Modells. Über die gesamte Untersuchung betrachtet zeigt sich, dass in fast allen Datensätzen Autokorrelation nach-

\footnotetext{
${ }^{8}$ Das $3 \sigma$-Intervall entspricht in etwa dem bereits erwähnten Signifikanzniveau von $95 \%$.
}

weisbar ist. Dabei führen (stark) positive $r_{\tau}$ der Zwischenankunftszeiten regelmäßig zu längeren Warteschlangen. Umgekehrt führen im Datensatz [E] negative $r_{\tau}$ zu kürzeren Warteschlangen. Eine Ausnahme bei dieser Verallgemeinerung sind einzelne Subdatensätze aus [A]. An dieser Stelle muss die Frage aufgeworfen werden, ob neben der Autokorrelation weitere Kennzahlen existieren, die Rückschlüsse auf das zu erwartende Systemverhalten zulassen.

\section{ZuSAMMENFASSUNG UND AUSBLICK}

Die vorliegende Arbeit untersucht den Einfluss von Autokorrelation auf Logistik- und Produktionsprozesse. Dazu werden Realdaten untersucht und in einem Simulationsmodell angewendet. Es zeigt sich, dass vor allem stark autokorrelierte Prozesse erheblich das Systemverhalten - hier die Warteschlangenlänge - beeinflussen können. Bemerkenswert ist auch, dass in fast allen vorliegenden Datensätzen signifikant Autokorrelation nachgewiesen werden kann. Dies alles führt zum Schluss, dass das Modellieren nach dem aktuellen Stand der Technik in der Logistik, d. h. dem Nichtbeachten von Autokorrelation bei der Prozessbeschreibung, zu Simulationsergebnissen führt, die nicht übertragbar auf die Realität sind. Folglich werden Zufallszahlengeneratoren notwendig, deren erzeugte Zufallszahlen die hier festgestellten Abhängigkeiten abbilden. Parallel muss aber weiter untersucht werden, ob neben Autokorrelationskoeffizienten noch andere, gegebenenfalls bessere Kennzahlen zur Charakterisierung von Daten existieren.

Die nächsten Schritte im Rahmen dieser Arbeit befassen sich mit dem Erzeugen von Zufallszahlen mittels der erwähnten Methoden. Diese sollen als Input von weiteren Simulationsstudien dienen und den Ergebnissen der Realdaten gegenübergestellt werden. Ziel ist es, identifizierte Prozesscharakteristika nachzubilden und entsprechende Systeme valide zu modellieren. 


\section{LITERATUR}

[Ach87] Achilles, M.: Zur Lösung der YuleWalker-Gleichungen. In: Metrika Bd. 34, Nr. 1, 1987, S. 237-251

[AM01] Altiok, Tayfur; Melamed, Benjamin: The Case for Modeling Correlation in Manufacturing Systems. In: IIE Transactions Bd. 33, Nr. 9, 2001, S. 779-791

[Ass00] Assenmacher, Walter: Induktive Statistik. Berlin, Heidelberg: Springer, 2000, - ISBN 9783540671459

[Ban00] Banks, Jerry: Getting Started with AutoMod. Bountiful: AutoSimulation Inc., 2000

[BJ70] Box, G. E. P.; Jenkins, Gwilym M.: Time series analysis: forecasting and control : Holden-Day, 1970

[CN96] Cario, Marne C.; Nelson, Barry L.: Autoregressive to anything: Time-series input processes for simulation. In: Operations Research Letters Bd. 19, Nr. 2 , 1996, S. 51-58

[DF79] Dickey, David A.; Fuller, Wayne A.: Distribution of the Estimators for Autoregressive Time Series with a Unit Root. In: Journal of the American Statistical Association Bd. 74, Nr. 366a, 1979, S. 427-431

[DH08] Dettling, Marcel; Hürzeler, Markus: Angewandte Zeitreihenanalyse. Zürich, ZHAW Zürcher Hochschule für Angewandte Wissenschaften, Vorlesungsmaterial, 2008

[Esh03] Eshel, Gidon: The Yule Walker Equations for the AR Coefficients. Chicago, University of Chicago, Working Paper, 2003

[Gla03] Glasserman, Paul: Monte Carlo Methods in Financial Engineering : Springer, 2003, -ISBN 9780387004518

[GMM88] Godfrey, L. G.; McAleer, Michael; McKenzie, C. R.: Variable Addition and Lagrange Multiplier Tests for Linear and Logarithmic Regression Models. In: The Review of Economics and Statistics Bd. 70, Nr. 3, 1988, S. 492-503
[JC85] Jain, Raj; Chlamtac, Imrich: The P2 algorithm for dynamic calculation of quantiles and histograms without storing observations. In: Communications of the ACM Bd. 28, Nr. 10, 1985, S. 1076-1085

[Ken53] Kendall, David G.: Stochastic Processes Occurring in the Theory of Queues and their Analysis by the Method of the Imbedded Markov Chain. In: The Annals of Mathematical Statistics Bd. 24, Nr. 3, 1953, S. 338-354

[Kud00] Kudlich, Thomas: Optimierung von Materialflußsystemen mit Hilfe der Ablaufsimulation. München, Technische Universität München, Dissertation, 2000

[Lec06] L'Ecuyer, Pierre: Uniform Random Number Generation. In: Henderson, S. G.; Nelson, B. L. (Hrsg.): Elsevier Handbooks in Operations Research and Management Science: Simulation. Amsterdam: Elsevier Science, 2006, S. 55-81

[LK00] Law, Averill M.; Kelton, David W.: Simulation Modeling and Analysis. 3. Aufl., Singapore: Mcgraw-Hill Professional, 2000, -ISBN 0071165371

[LM91] Lewis, P. A. W.; McKenzie, E.: Minification processes and their transformations. In: Journal of applied probability Bd. 28, Nr. 1, 1991, S. 45-57

[LMN90] Lucantoni, David M.; Meier-Hellstern, Kathleen S.; Neuts, Marcel F.: A single server queue with server vacations and a class of non-renewal arrival processes. In: Advances in Applied Probability Bd. 22, Nr. 3, 1990, S. 676-705

[LMT93] Livny, Miron; Melamed, Benjamin; Tsiolis, Athanassios K.: The Impact of Autocorrelation on Queuing Systems. In: Management Science Bd. 39, Nr. 3, 1993, S. 322-339

[Mad08] Maddah, B.: Random-Number Generators. Beirut, American University of Beirut, Vorlesungsmaterial, 2008 
[Mel91] Melamed, Benjamin: TES: A Class of Methods for Generating Autocorrelated Uniform Variates. In: ORSA Journal on Computing Bd. 3, Nr. 4, 1991, S. $317-$ 329

[Nie04] Nielsen, Hejn Erland: Streams of events and performance of queuing systems: The basic anatomy of arrival/departure processes, when focus is set on autocorrelation. In: CORAL Working $\mathrm{Pa}$ pers Bd. L-2004, Nr. 02, 2004, S. 1-21

[Nie07] Nielsen, Hejn Erland: Autocorrelation in queuing network-type production systems-Revisited. In: International Journal of Production Economics Bd. 110, Nr. 1-2, 2007, S. 138-146

[PDM93] Patuwo, Eddy B.; Disney, Ralph L.; McNickle, Donald C.: The effect of correlated arrivals on queues. In: IIE Transactions Bd. 25, Nr. 3, 1993, S. $105-110$

[Run62] Runnenburg, J. Th.: Some numerical results on waiting-time distributions for dependent arrival-intervals. In: Statistica Neerlandica Bd. 16, Nr. 4, 1962 , S. $337-347$

[Sim12] SIMUL8 Corp.: SIMUL8 Knowledge Base: What Random Number Generator does SIMUL8 use? URL http://support.simul8.com. - abgerufen am 24.07.2012

[Sk173] Sklar, Abe: Random variables, distribution functions, and copulas. In: Kybernetika $\mathrm{Bd}$. 9, Nr. 6, 1973, S. 449-460

[SS01] Schlittgen, R.; Streitberg, B.H.J.: Zeitreihenanalyse. 9., unwesentlich veränderte. Aufl., München, Wien: Oldenbourg Wissenschaftsverlag, 2001

[VDI10] VDI 3633: Simulation von Logistik-, Materialflu $\beta$ - und Produktionssystemen; Grundlagen. Berlin: Beuth Verlag, 2010

[YAG05] Yücesan, E.; Andradottir, S.; Goldsman, D.; Schruben, L.W.; Schmeiser, B.W.: Analysis methodology: are we done? In: Proceedings of the 2005 Winter Simulation Conference. ACM, Orlando, 2005, S. 790 - 796
[Zae02] Zäh, M.; Reinhat, G. (Hrsg.): Fabrikplanung 2002 - Visionen, Umsetzung, Werkzeuge, iwb Seminarberichte. München: Herbert Utz Verlag, 2002 ISBN 9783896750631 